\title{
Self-Healing Besides Restoration Methods in Smart Grid Distribution Networks
}

\author{
Korra Cheena
}

\begin{abstract}
The Smart grid refers to a next-generation power grid which is a two-way information flow where electricity and information switch over between the service and its customer's. The power system becomes smart by applying intelligence by way of multidirectional flow of electricity and information to create an extensive distribution network through smart grid technology. It made smarter power system by developing a networking communication, controls, automation, new technologies and tools working together to make the great efficient and more secure environment. For an effective integration and quality of the service to the consumer's smart grid technology is needed due to working with the electrical distribution system and quickly to respond digitally for rapidly changing electric demand. At this point, an electricity disruption such as a blackout can affect a series of failures that can affect several areas such as banking, traffic, and security. To address the power restoration, smart grid makes use of self-healing strategy which will allow automatic switching when equipment failure or outages occur. There have been numerous studies in the last decade or so in to even out the fundamental and mathematical challenges of making a smart self-healing grid a reality. In this paper, we explore the Selfhealing approach for Smart grid Communications likewise discusses the service restoration methods in Distribution Networks of Smart Grid Environment.
\end{abstract}

Keywords: Smart grid, Power system, Power restoration, Selfhealing. FLISR, Meta-heuristic algorithms

\section{INTRODUCTION}

A community like a residential, commercial business customer such as banking, traffic, hospitals, railways, data centres and security needs uninterrupted power supply and all these are connected with nearby distribution substations. Due to lightning, failure of devices and limitations, overheating and natural accidents in the distribution region may cause the power outage without any kind of prior information. A self-healing system is very much required in the smart grid distribution networks for providing uninterrupted power supply to all these emergency services. The present power grid management system could not identify the fault and power restoration in the network in time which commits huge loss to the consumers. In this connection self-healing system needs to use more efficient sensing components in the distribution system. The wireless sensor network based smart grid technologies deploy sensors at power substation, and in power lines to detect the problem and communicate the same with the control system and to the base station. The disruption and minimized control system continuously monitors the state of the grid.

Revised Manuscript Received on April 30, 2020.

* Correspondence Author

Korra Cheena *, assistant professor and head of department in Electrical \& Electronics Engineering, University College of Engineering, Kothagudem. Email: korrachinna@gmail.com

(C) The Authors. Published by Blue Eyes Intelligence Engineering and Sciences Publication (BEIESP). This is an open access article under the CC BY-NC-ND license (http://creativecommons.org/licenses/by-nc-nd/4.0/)

The control switches automatically isolate the faulted section from the power line and finds the best way to restore the power into the network automatically using control system reconfiguration. A WSN based closed loop distribution network will provide uninterrupted power supply to consumers. The sensor nodes may have the following features in the distribution system

1. More information about the state of the grid.

2. Continuous grid assessment using control algorithm.

3. Enhanced restoration system could perform rapidly to detect the fault.

1.1 The Self-Healing Grid: This system encompasses with sensors programmed controls, and cutting-edge software that deploys real-time data distribution to identify and isolate faults and to reconfiguration of the distribution network to reduce the customer impacted.

1.2 Goals of Self-Healing Grid: The primary purpose of the self-healing in power delivery systems is to improve system reliability by quick identification and isolation of faulted system components and the restoration of service to customers as possible from alternate sources/feeders above activity achieved with or without human intervention while restoring the system. Self-Healing of power distribution systems is lead via Distribution Automation (DA), precisely over smart protective and switching devices that minimize the number of interrupted consumers during likelihood situations by inevitably separating faulted components and relocating customers to an optional source when their normal supply has been lost. Distribution Automation system relating by way of the substation, feeder and customer automation, this is the possible things to motivating the smart grid for consistency and power quality, controlling incentives and penalties, the pressure to cut costs and optimize operations by minimization of time to reconfigure and restore service.

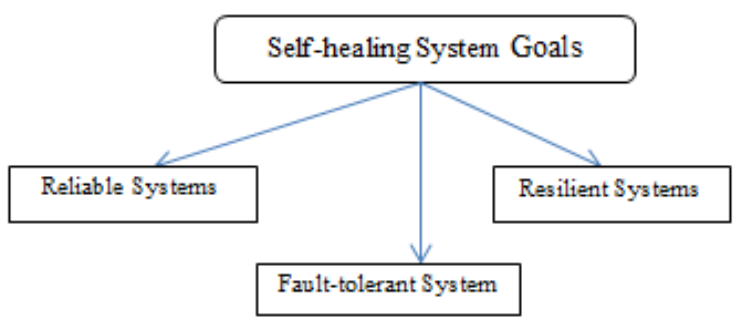

Fig. 1. Self-healing system goals

Published By: 


\section{Self-Healing Besides Restoration Methods in Smart Grid Distribution Networks}

\section{MOTIVATION}

One of the major challenges for distribution networks is the ability to deliver high quality and reliability to customers. An intelligent distribution network must consider that self-repairing control methods meet the above requirements. Compared to the traditional distribution network, the intelligent distribution network must achieve self-regulation control related to individual detection of errors, troubleshooting and location acceleration, recovery of errors and energy from the fault lines. The rapid detection of various failures, the accurate identification of defective components, and the precise differentiation of these defective components are the basis and the need for selfregulatory control functions in automated distribution networks. In addition, maximum restrictions such as partitioning and recovery of services from (i) blocked locations of the distribution network must be respected; (Ii) Limit the minimum number of switching operations.

\section{SELF-HEALING APPROACH FOR SMART GRID COMMUNICATIONS}

In Smart grid information from across the grid i.e. two-way communication reaching inside the home similarly, the information could be collected from the advanced sensors and metering, customers are motivated to manage the load, and few advanced applications could support decision making through system analysis as shown in figure 3.

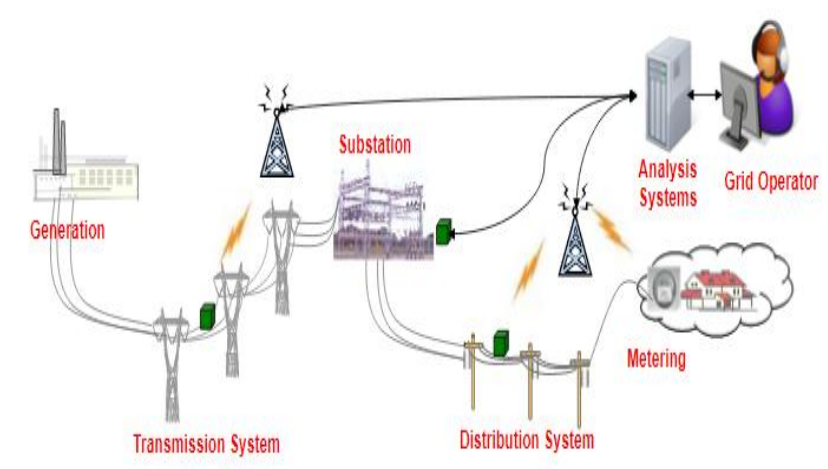

Fig. 2.The Smart grid Communications system

There is a potential Self-healing approach to improve distribution system reliability i.e. FLISR (fault location, isolation, and service restoration) which is being implemented by many utilities is a potentially powerful capability.

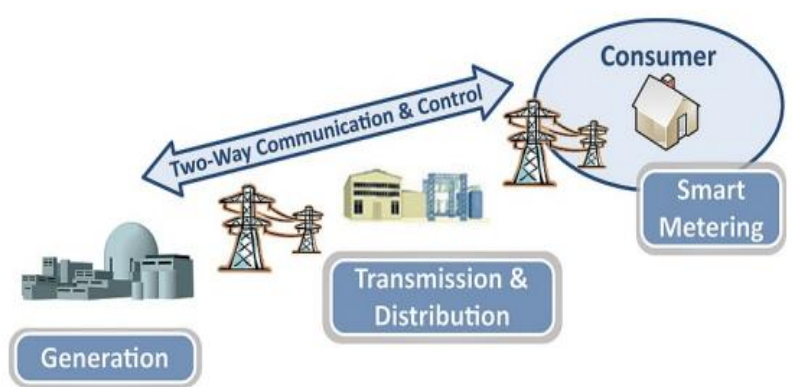

Fig. 3. Two-way Communication Smart grid
The basic idea of FLISR is to identify the fault area as quickly as possible and to isolate the fault area as closely as possible, which means that the impact of a power failure due to failure (in terms of duration and number of customers or load). FLISR is called "self-healing" network capacity. And while it is not completely self-healing, the goal is to help ensure a more optimal network configuration at lower optimum operating conditions. It therefore requires an accurate grid layout; Precise information on network operating conditions, a certain level of remote switching power and optimization of algorithms for optimizing imperfect conditions.

\subsection{Fault Location}

The initial step in the FLISR process is the fault location. Locating the fault is a prerequisite to all future actions and, with many utilities, an opportunity to improve reliability regardless of the level of automation available to support fault isolation and grid reconfiguration.

Faults on the distribution system can be caused by a variety of factors from equipment or cable failures, structural damage to facilities from weather-related events, and animal encroachments. In some cases, where physical damage to the system is reported by customers, identifying the source and location of the fault can be accomplished quickly. However, in many cases, the source and location of the failure are not apparent, and service restoration activities are delayed until the fault location can be identified. Faults on underground systems can be especially challenging to locate since physical inspection at those locations is extremely difficult or impractical.

Utilities have employed a variety of methods and technologies to assist with fault location. Some of these involve advanced technologies, while others are highly manual and time-consuming. Commonly used methodologies can generally be grouped into one of the following three categories:

1. Portable test equipment such as cable thumpers or Time Domain Reflectometry (TDR) devices typically used to support underground fault location

2. Permanently installed line indicators - either noncommunicating visual indicators or communicating sensors installed on overhead or underground feeders

3. Permanently installed equipment at substations or operations centers.

- Protective relays and digital fault recorders.

- Voltage drop triangulation methods.

- Impedance-based fault location methods.

\subsection{Fault Isolation}

The goal of the fault isolation component of FLISR is to minimize the impact of an outage through a reconfiguration of the distribution grid where this impact can be evaluated in several ways. Widely used metrics include the total number of customers or the whole load affected by an outage. Today's ADMS(expand) solutions typically support reconfiguration, or grid optimization, capability. 
However, other factors, such as critical loads affected, may need to be taken into consideration as well. With the proliferation of distributed energy resources (DER) in some service areas, the impact of circuit reconfiguration on DER operation must be considered, as well as the protection scheme and worker safety implications. The latter two are related to the two-way nature of power flow in the distribution system when DER is permitted to inject into the system, and this must be taken into account when the isolation and reconfiguration/optimization is employed. With these kinds of considerations, fault isolation/grid reconfiguration becomes increasingly complex, and higher levels of optimization capability are required.

In addition to identifying the optimal grid reconfiguration for an identified fault, the distribution management system must be able to eliminate potential grid reconfigurations that are not supportable. Therefore, before an optimal solution can be implemented, time-forward simulations should be performed to evaluate future loading and DER production to ensure that the new system configuration (and any intermediate steps required to achieve this configuration) will not result in violations during the forecasted period.

As with fault location, there are many different approaches to fault isolation and circuit reconfiguration among utilities. Many utilities have implemented decentralized fault detection and isolation schemes that react in real-time to a detected fault. These decentralized schemes can be expected to provide an immediate grid reconfiguration that narrows the impact of an outage. However, it is more common for utilities to deploy centralized grid management applications (protective relaying, ADMS, DMS/OMS) for FLISR capabilities. The centralized capability may be in addition to decentralized schemes, requiring some level of coordination and awareness at the centralized level. While some utilities have adopted fully automated FLISR operations, others adopt more semi-automated schemes, with the final reconfiguration action dependent on operator review and manual switching operations.

\subsection{Service Restoration}

The reconfiguration associated with isolating the fault really begins the service restoration process by narrowing the extent of the outage and returning more customers to service. The remainder of the service restoration process remains largely manual. However, the fault location process can be expected to accurately pinpoint where the repair crews need to focus their efforts. This is especially valuable on underground circuits where, otherwise, a time-consuming and expensive process to locate the source of the fault would likely be required before repairs can begin. With the integration between the fault location application and the work management application, the coordinates for and/or a map of the likely fault location can be transmitted to the field crew. This, together with infield access to an "as-built" GIS map of the grid can speed the restoration process. In-field access to the GIS map also provides the ability for the field crew to update configuration/circuit information back into GIS postrestoration - maintaining the alignment between the physical grid and the grid model.

FLISR has many faces, in that it can encompass various technologies and different approaches to realize its

goal of minimizing the outage impact of faults on customers. With good choices, the potential benefits of a successful FLISR capability can be significant relative to its cost. In developing a roadmap and business case for FLISR, utilities should consider how the full range of today's technologies (as well as existing data and infrastructure investments) can be leveraged to improve each aspect of fault location, isolation, and service restoration.

\section{SERVICE RESTORATION METHODS IN SMART GRID}

The European Energy Regulators Council [19] defines a smart grid to control the spread of the smartest and best used technologies and solutions and to implement the existing grid so that the spirit of a new grid is better planned and launched. Services and CE Capacity Improvement. "Author "focus was on to prepare the solution or the technology, which makes better utility for the user community. The evolution of the technology for faster communication has made it possible to catch the interest in terms of controlling the systems in the real-time. Hence, the characteristics of the smart grid have to be exploited to increase its efficiency.

The essential functions or tasks that are connected to the ongoing work are an assessment of real-time data, controlling the equipment's in the real-time, and distribution of the load to satisfy the needs. Intelligence is required for the processes which handle the above tasks called intelligent electronic device (IED), which, however, does not have a proper definition.

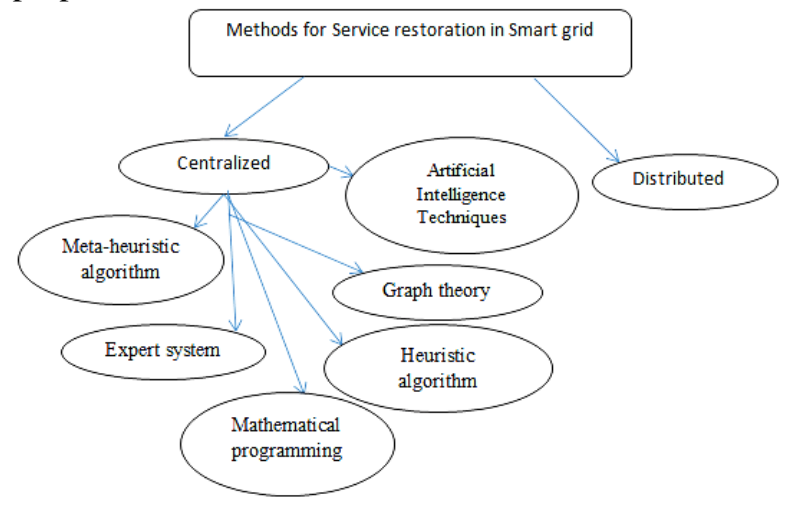

Fig.4: Hierarchical structure for Service restoration

To address the complexity, the concept of IED can be simplified as the device possessing communication and control capabilities. These devices can maintain various functions such as protection, data logging and metering. But more focus is on to limit the services to control and communication. The advantage of the control center is that it possesses the capability to receive the real-time data continuously, and the center optimizes the network's condition all the time. If, in the current network conditions, any modifications are required then these modifications are communicated to the IED which attends to change the switch condition of the current network at the particular node. The present day's smart grid requires that it shall be ready for extensively distributed generation, apart from the actual implementation and research. 


\section{Self-Healing Besides Restoration Methods in Smart Grid Distribution Networks}

The author, in the current work, has not attended the detailed modeling for the distributed generation source. The author's consideration was limited to the negative load at the bus. It is an approximation to develop comfort for the computation to generate a faster solution. This solution makes the more rapid response which further produces the benefit of optimization to achieve high reliability by minimizing the network condition. In the case of faults or changes in the main load then a process would be initiated to reconfigure the network. From the proposed algorithm modification in the network can be achieved for the efficient functioning of the system.

One of the challenging problems is the restoration, which is complicated in the case of the systems which practically large. The solution for the restoration problem, which was proposed by the researchers, includes many techniques viz. mathematical programming, heuristics, etc.

\subsection{Mathematical Programming}

Logically, the restoration problem is related to optimization. Hence the mathematical programming techniques become the direct solution techniques for this restoration problem. One of the mathematical search techniques was used to find the solution for the challenge. In the process of finding the optimal solution for the restoration problem, mathematical problem offers the advantage. Though the search space is the dependency factor for the answer, hence, the efficient distribution systems which are large, the time required for the solution might be long enough. Because of the complicated scenario of the practical distribution networks, to formulate the mathematical solution in a considerable time, every operational characteristic cannot be considered. It is observed that the research carried out, in the direction of the usage of mathematical programming to provide the solution for restoration problem, is little. The fundamental problem the researchers desired to tackle in the current research is to enhance the time limit is to simplify the mathematical solution. An investigation was carried out in [1] towards converting the non-linear reconfiguration problem into second-order cone problem for the ease in manipulation. But it is found that it the processing time needs to be considered to be enhanced in the case of large systems.

\subsection{Heuristics}

To solve the problem, the heuristic techniques take divide-and-conquer as the base and experiences into consideration. Such techniques cannot assure the optimal solution; however, those solutions are acceptable for the given time [2]. The primary characteristic of any heuristic technique is that it uses intellectual shortcuts to assist the solution developers in finding a quick solution. Some of the examples for heuristic techniques are common sense, refined speculation, and thumb rule. Another characteristic of heuristics is they are smarter when compared with the conventional optimization techniques. It is because optimization techniques require more computation, whereas heuristics reduce that burden [3]. However, heuristics to carry disadvantages such as producing the solutions with poor confidence in a few scenarios. Hence, designing the heuristic techniques for a solution ought to be careful to assure a near-optimal solution. Such a solution is implementable in a practical network by the operational engineers. The capability for restoration is significant for a quick load which is a substantial benefit when heuristics are used. From various researches, it has been observed that there was extensive usage of heuristic techniques to solve the restoration problem [4-5]; however, it is uncertain about solving optimal restoration problem. Furthermore, the number of switching operations required are enormous; in some scenarios [6]. Considerable efforts are expended in the process of performance enhancement when heuristics are used to develop the solutions, especially with the combination of fuzzy set theory [7].

\subsection{Meta-heuristic algorithms}

Among the family members of the Meta-heuristics, there are many evolutionary algorithms such as genetic algorithms, swarm optimization which is one of the naturally self-conscious algorithms, simulated annealing, along with the search which one of the local search methods. In the process of developing solutions for the restoration problem, meta-heuristics were successful. These algorithms are ideal for restoration problem, which is one of the hard optimization problems. The property of the evolutionary algorithm is population-based and meta-heuristic optimization algorithm.

The inspiration for the techniques used in evolutionary algorithms is biological changes: mutation and reproduction. The initial population is the initiating process for the solution, which then fitness function would decide the successive evolution of the population. To reach the final solution, the process involves several evolution operations. One of the familiar evolutionary algorithms, genetic algorithm, imitates the human genetic process to discover the near-optimal solution. Adoption of the genetic algorithm is found in several research presentations for the relevance in producing the solutions for similar optimization problems, especially restoration problem [8-9]. Differential evolution, evolution strategy $[10,11]$ is the other evolutionary techniques which are used to attend for solving the service restoration problem. However, in other researches, several different naturally self-conscious algorithms are used to solve restoration problem, among which particle swarm optimization was found successful in [12]. The technique used in particle swarm optimization to solve the optimization problem is, bird flocking. The solution was established by simulating the bird flocking in the 2-dimensional space. The key optimization factors are the velocity and position of each agent. Any change in the position of the agent is realized from the information produced through velocity and position. It is already observed the success of local search methods, and the positive role of meta-heuristic algorithms in solving the large systems. But the greatest concern is the computation time in the scenarios of large networks. Besides, the computation time would be affected by the data structures used for meta-heuristic algorithms. Hence, data structures shall be chosen carefully to improve the performance of the system in terms of computation time though the metaheuristic algorithms develop a near-optimal solution but cannot be optimal. 
However, it is found that heuristic solutions are better and are accepted by several operation engineers.

\subsection{Expert systems}

Prerequisites for the techniques based on expert systems are operational variables and comprehensive knowledge of the system. Data is gathered, expert rules are formulated for the system in the process building an expert system to solve the problem. Hence, it can be interpreted that the expert system imitates the human expert system's decision process. Heuristics support the expert systems in such close association that ES are misinterpreted as heuristics. Not much research is found in the recent works in the area of restoration problem by using expert systems [1314]. Expert systems capability to find a faster solution in restoring the system is through the combination of expert rules and database. But the basis for the expert systems is distribution network's technical data stored in the database, and the expert rules. Hence, techniques used for expert systems are discouraged due to the system's dependency, which further generates a question about the suitability of the methods [15]. Expert systems too, do not assure for the optimal solution as is the case with heuristics [16].

From $[17,18]$, it is found that fuzzy set theory and artificial neural networks (ANN) are useful in the solution for restoration problem. The advantage of fuzzy logic is that it is possible to represent the human experience wherein the properties of inputs are fuzzy [16]. In solving the restoration problem, with the help of a predetermined solution, ANNs would be trained. Such techniques act fast but are found dependent on the system, required a large amount of training data. Moreover, they do not assure the optimal solution.

\subsection{Graphs Theory}

A distribution network can be understood as a graph representing trusses in the form of nodes and lines connecting trusses to the edges of the graph. As a result, the problem of rebuilding the service can be seen as the problem of finding a large tree that represents the radial structures of the network and meets the operating restrictions.

\section{RESULT ANALYSIS}

Objective to Restore Active Distribution Network Service with Mathematical Model DG: Connectivity and Radial Topology to Meet Demand, Feeders, Load Transformers and Switches After Service Restore Not Overloaded With Minimal Switch Operation, Low Power Down Time, Minimum Network Loss, Too Much Load. Stable and stable Reliable, load balancing, maximum power limit.

\section{Priority in load restoration}

Different loads require different feed reliability loads are divided into two priority levels to regain maximum power at the same time, ensuring that high priority loads are first restored. The objective function is as follows:

$\max f_{1}(x)=\sum_{i=1}^{n} A_{i} S_{i} P_{i}$ $\max f_{2}(x)=\sum_{i=1}^{n} A_{i} S_{i} Q_{i}$

The total number of affiliates involved in service renewal is $n ; x$ is the current network state associated with the closed state of all affiliates involved in service renewal; In the closed position of switch number $i$, where "0" means open and "1" is closed; $\mathrm{Ai}, \mathrm{Pi}$ and Qi are weighted coefficients, preferably with the node load, the nominal reactive power and the nominal reactive power associated with branch number i. To determine the highest priority level load in the power supply,, the weight of the top layer is greater than the weight of the second priority level.

\section{The minimum counts of switching operation}

$\min \mathrm{N}=\sum_{i=1}^{n}\left|K_{i}-S_{i}\right| \Rightarrow \max \mathrm{f}_{3}(\mathrm{x})=\frac{1}{N}$

Where $K_{i}$ is the initial closed state of the branch numbers $i$; $\mathrm{S}_{\mathrm{i}}$ is the closed state of the switch after restoration; $\mathrm{N}$ is the number of switch operation.

\section{As far as possible to reduce the line loss after service restoration}

Line loss directly affects the economic operation of the distribution network, in order to improve the economic benefit of the service restoration; we should reduce the line loss as much as possible.

$$
\min \sum_{j=1}^{n_{1}} P_{\text {sun }(j)} \Rightarrow \max \mathrm{f}_{4}(\mathrm{x})=\frac{1}{\sum_{j=1}^{n_{1}} P_{\operatorname{sun}(j)}}
$$

Where $P_{\text {sun }(j)}$ is the active power loss of the branch number $\mathrm{j} ; n_{1}$ is a collection of all branch in the network.

Maximize the power supply of the distribution generation

$$
\max f(x)=\frac{1}{\sum_{i \in C}\left(P_{G \max }^{i}-P_{G}^{i}\right)}
$$

Where $P_{G \max }^{i}$ expresses maximize the power supply of the generation $\mathrm{i}$.

Distribution network with DGs is shown in figure 5.

Table 1. Installation position and capacity and the type of DGs

\begin{tabular}{|l|l|l|l|l|}
\hline $\begin{array}{l}\text { DG } \\
\text { Number }\end{array}$ & $\begin{array}{l}\text { Installation } \\
\text { node }\end{array}$ & $\begin{array}{l}\text { Rated } \\
\text { Capacity }\end{array}$ & Capacity & $\begin{array}{l}\text { DG } \\
\text { Type }\end{array}$ \\
\hline 1 & 10 & $200 \mathrm{~kW}$ & $100 \mathrm{~kW}$ & NBDG \\
\hline 2 & 15 & $200 \mathrm{~kW}$ & $70 \mathrm{~kW}$ & BDG \\
\hline 3 & 23 & $200 \mathrm{~kW}$ & $90 \mathrm{~kW}$ & NBDG \\
\hline 4 & 32 & $200 \mathrm{~kW}$ & $100 \mathrm{~kW}$ & BDG \\
\hline
\end{tabular}




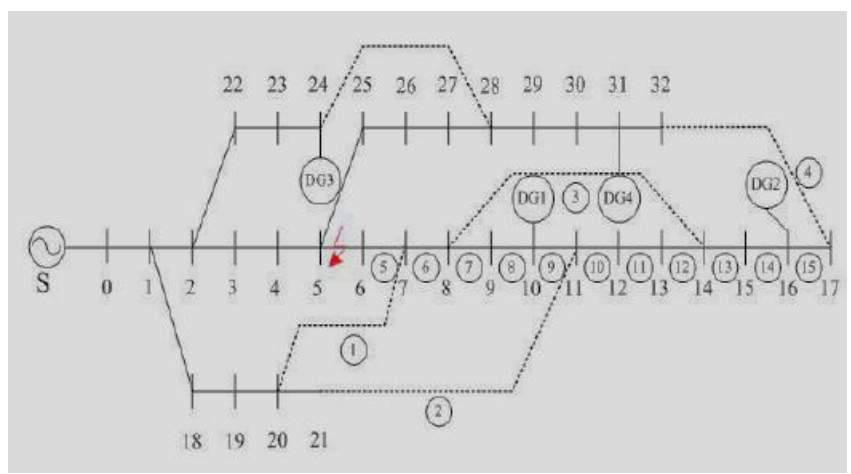

Figure 5. 33 nodes distribution network with DG

\section{RECOMMENDATIONS FOR FUTURE RESEARCH}

In traditional grids, system reconfiguration for service restoration is a potential problem, even though it was addressed by smart grid context. Still, there are many issues to be considered for optimal solutions, and novel features are required for the quick restoration process. Some of the points are suggested for future research.

- An effective two-way communication system should be built for communication among control centers and automatic switches for on/off commands and response of automatic switches.

- There is a research need on the upgrading of mathematical techniques to minimize the restoration time over an extensive system.

- Need to focus on graph theory to address the restoration problem in the smart grid environment.

- There is a scope of the research in the area of decentralized control approaches for service restoration in a smart grid environment.

\section{CONCLUSION}

In this paper, we have described the Self-healing system goals that are based on power delivery systems to improve system reliability by the quick identification and isolation of faulted system, advanced stochastic optimization techniques and theory, and experience of power engineering to address the system restoration problem in large-scale power systems. We referred to as "self-healing" approach FLISR as fault location, isolation and service restoration. And, although, not fully self-healing, the goal is to help ensure a more optimal smart grid configuration under suboptimal operating conditions. Furthermore, we explore the

Service Restoration Methods for Smart grid environment and recommend a few future research directions for optimized self-healing system for smart grid network.

\section{REFERENCES}

1. D.S. Sanches, J.B.A. London Jr, A.C.B. Delbem, R.S. Prado, F. G. Guimara es, O.M. Neto, T.W. de Lima, Multiobjective evolutionary algorithm with a discrete differential mutation operator developed for service restoration in distribution systems, Int. J. Electr. Power Energy Syst. 62 (2014) 700-711.

2. P.M.S. Carvalho, L.A.F.M. Ferreira, L.M.F. Barruncho, Optimization approach to the dynamic restoration of distribution systems, Int. J. Electr. Power Energy Syst. 29 (2007) 222-229.

3. K.N. Miu, C. Hsiao-Dong, R.J. McNulty, Multi-tier service restoration through network reconfiguration and capacitor control for large-scale radial distribution networks, IEEE Trans. Power Syst. 15 (2000) 1001-1007.
4. T. Nagata, H. Sasaki, A multi-agent approach to power system restoration, IEEE Trans. Power Syst. 17 (2002) 457-462.

5. T. Yokoyama, T. Nagata, A multi-agent restoration method for the distribution network, Energy Procedia 14 (2012) 726-731.

6. S. Dimitrijevic, N. Rajakovic, An innovative approach for solving the restoration problem in distribution networks, Electr. Power Syst. Res. 81 (2011) 1961-1972.

7. M.A. Pereira, C.A.F. Murari, C.A. de Castro Jr, A fuzzy heuristic algorithm for distribution systems' service restoration, Fuzzy Sets Syst. 102 (1999) 125-133.

8. H. Ying-Tung, C.Y. Chien, Enhancement of restoration service in distribution systems using a combination fuzzy-GA method, IEEE Trans. Power Syst. 15 (2000) 1394-1400.

9. R.E. Brown, A.P. Hanson, Impact of two-stage service restoration on distribution reliability, Power Syst., IEEE Trans. 16 (2001) 624-629.

10. R.S. Prado, R.C. Pedrosa Silva, O.M. Neto, F.G. Guimara es, D.S Sanches, J.B.A. London Jr, A.C.B. Delbem, Differential evolution using ancestor tree for service restoration in power distribution systems, Appl. Soft Comput. 23 (2014) 498-508.

11. A. Augugliaro, L. Dusonchet, E. Riva Sanseverino, Multiobjective service restoration in distribution networks using an evolutionary approach and fuzzy sets, Int. J. Electr.Power Energy Syst. 22 (2000) 103-110.

12. A.M. El-Zonkoly, Power system single step restoration incorporating cold load pickup aided by distributed generation, Int. J. Electr. Power Energy Syst. 35 (2012) 186-193.

13. K. Hotta, H. Nomura, H. Takemoto, K. Suzuki, S. Nakamura, S. Fukui, Implementation of a real-time expert system for a restoration guide in a dispatching centre, in Power Industry Computer Application Conference, Seattle, WA, 1-5 May 1989.

14. A.A. Hafez, W.A. Omran, Y.G. Higazi, A decentralized technique for autonomous service restoration in active radial distribution networks, IEEE Trans. Smart Grid 9 (3) (2018) 1911-1919.

15. S. Dimitrijevic, N. Rajakovic, An innovative approach for solving the restoration problem in distribution networks, Electr. Power Syst. Res. 81 (2011) 1961-1972.

16. T. Yokoyama, T. Nagata, A multi-agent restoration method for the distribution network, Energy Procedia 14 (2012) 726-731.

17. H.-T. Yang, C.-M. Huang, Distribution system service restoration using fuzzy Petri Net models, Int. J. Electr. Power Energy Syst. 24 (2002) 395-403.

18. C.-M. Huang, C.-T. Hsieh, Y.-S. Wang, Evolution of radial basis function neural network for fast restoration of distribution systems with load variations, Int. J. Electr. Power Energy Syst. 33 (2011) 961968.

19. U.S. Department of Energy, Fault Location, Isolation, and Service Restoration Technologies Reduce Outage Impact and Duration, December.

\section{AUTHOR PROFILE}

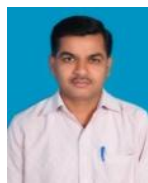

Korra Cheen received his $M$. Tech at NIT Calicut, KERALA. He is pursuing his Ph.D at IIT Dhanbad. He is working as an assistant professor and head of department in Electrical \& Electronics Engineering at University College of Engineering, Kothagudem. His areas of interests are Control Systems, Digital Control Systems, Smart Grids,

Power System Operation \& Control, and Power Electronics. 